\title{
NOTAS S OBRE LAS ACUMULACIONES DE LADERA EN EL MACIZO DEL MAIGMÓ
}

\author{
Juan Antonio Marco Molina
}

\section{RESUMEN}

En este estudio se analizan las acumulaciones de ladera del Macizo del Maigmó (Alicante), formas del modelado encargadas de la regulación de las vertientes. Para ello se estudia la relación entre las laderas cantil-talud y las morfoestructuras, distinguiéndose dos tipos de laderas. En los depósitos hay una clara diferenciación entre los canchales (actuales o subactuales) y acumulaciones más antiguas (pleistocenas). En los primeros, además de diferenciar varios tipos según su morfología perimetral, se analizan los perfiles para determinar su naturaleza; en franco contraste, las acumulaciones infrayacentes completan la morfología de estas laderas.

\begin{abstract}
This study analyses the slope debris of the Maigmó mountain (Alicante), forms which are in charge of regulating these slopes. The relation between the morpho-structure is studied making a distinction between two different types of slopes. There is a clear difference between the present day screes or these belonging to a near past and more ancient deposits (Pleistocene). Amongst the first, apart from distinguishing several types depending on the perimetral morphology, we analyse the section in order to determinate the nature; on the other hand the deposits which lie underneath complete the morphology of these slopes.
\end{abstract}

En el modelado de las vertientes del ámbito estudiado, se ha concedido especial interés a aquellas cuya evolución parte de la dialéctica morfográfica existente entre los cantiles y los taludes que se desarrollan en sus sopiés.

En trabajos anteriores ya se han analizado los procesos y parte de las formas 
resultantes ${ }^{1}$, pero la continuación de esta línea de trabajo, y los numerosos trabajos y recorridos de campo que se efectúan, obligan a revisar o acendrar opiniones e interpretaciones pretéritas, con lo que se pretende un conocimiento cada vez más preciso de la realidad. El objetivo del presente estudio pues, no es otro que ampliar y perfeccionar el conocimiento de las acumulaciones de ladera que escapan al calificativo estricto de fluviales.

Sin embargo, es obligado matizar la denominación de «acumulaciones», pues si el estatismo es un rasgo inherente a la misma, no hay que descartar la tendencia a la movilidad de buena parte de las laderas en las que se ubican. Son por tanto laderas que han evolucionado, y en cada momento de la evolución han resp ondido a ambientes diferentes hasta llegar al momento actual o subactual.

\section{Laderas cantil-talud y control estructural}

La distribución espacial de este tip o de laderas y p or ende del objeto de estudio, tiene una estrecha relación con la localización de las fallas. Son pues vertientes en las que su morfoestructura es consecuencia de una tectónica de fractura que ha afectado a la cobertera sedimentaria y ha determinado top ografía, altimetría y rep arto de los materiales de características físicas bien contrastadas.

La consecuencia más directa ha sido la elevación de los materiales calcáreos (cantiles) que dominan otros, infray acentes, may oritariamente margosos. También se da el caso de que el cantil domine un talud compuesto únicamente por materiales detríticos cuaternarios, ya que no afloran materiales margosos, bien porque el salto de falla no es bastante alto, bien porque la inclinación de las calizas o las características de las fallas introducen matices estructurales que hacen variar no sólo el esquema de la ladera, sino también la morfología de los sedimentos.

De esta manera podemos distinguir dos conjuntos de laderas: unas con calizas suavemente inclinadas sobre margas o materiales más blandos, en ellas hay una clara diferenciación morfográfica entre el cantil y el talud; y por otro lado, aquellas en las que la may or inclinación, incluso hasta la vertical, impide que la ruptura de pendiente entre el cantil y el talud sea tan clara.

Al primer conjunto señalado pertenecen amplios sectores de todo el macizo cuya percepción no es difícil, pues se trata de grandes alineaciones de escarpes de línea de falla que sin solución de continuidad aparente (existen pequeñas desnivelaciones motivadas por fallas o fallas-desgarres transversales que accidentan la unidad), se extienden varios kilómetros, de forma rectilínea unas veces, con pequeños esconces otras.

De norte a sur incluimos dentro de este conjunto el escarpe septentrional de la Serra de l'Arguenya o Carrascal de Castalla, con El Puntal como bloque hundido y más avanzado hacia el norte que domina con un desnivel de $200 \mathrm{~m}$ el sector occidental del valle de la Arguenya; se trata de una ladera compuesta por las calizas eocenas, y el talud lo constituyen las margas eocenas y paleocenas, con un buzamiento general hacia el sur. 
El escarpe tendido de norte a sur desde la Serra de Castalla o Serratella hasta el Despenyador que destaca entre 460 y 720 m sobre el valle del Riu Verd-Montnegre-Sec, la continuación del mismo escarpe hacia el SO en la Serra del Frarel; en ambos casos constituyen el cantil las calcarenitas serravallienses, mientras que el talud lo integran las margas blancas del Langhiense materiales que en primer tramo señalado buzan hacia el oeste, y después en la Serra del Frare hacia el norte.

En el mismo tipo incluimos la continuación de la alineación del Maigmó hacia el oeste en su tramo más próximo al Estret, y en las Peñas del Señor, ambas con buzamiento invertido, buzando hacia el sur, y con el cantil constituido por calizas cenomanienses y turonienses.

Frente a estas laderas que acabamos de señalar están aquellas que se incluyen en el segundo conjunto, cuya característica principal es la de no mantener de forma tan tajante la contraposición morfológica entre el cantil y el talud, es decir, las características morfoestructurales -o morfográficas si se quiere- de estos relieves impide una diferenciación tan nítida de ambos elementos, ya que en no pocas ocasiones ap arecen solapados.

El rasgo más llamativo de alguna de estas vertientes, es el que no suponen unidades top ográficas de dimensiones comparables a las anteriores, aunque no por ello escapen a la percepción, todo lo contrario, les confiere un cariz señero, singular que los hace resaltar. Se hace referencia a aquellas elevaciones, picos que pueden acabar en pequeños cuetos o afiladas agujas. En otros casos, a pesar de tener may or continuidad espacial, son crestas en las que las calizas del cantil están muy inclinadas incluso verticales, y con frecuencia doblan el esquema del cantil.

En este conjunto incluimos el frente oeste del Maigmó p rop iamente dicho con las calizas turonienses en su cúspide y la serie senoniense en la base con un buzamiento invertido, hacia el sur; también hay que considerar aquí gran parte del frente meridional del mismo, en el que las cap as calizas, cortadas a cercén o en pequeñas viseras, son una sucesión continua de pequeños cantiles muy triturados, en un frente en el que no faltan pequeñas fallas transversales.

El frente norte de la alineación del Maigmó que domina el valle de línea de falla desde el Collado d'Exaú hasta el Estret prácticamente con un desnivel entre 200 y 150 m. Las Peñas Montesas, en ambas vertientes, con el doble cantil de las calizas cenómano-turonienses verticales; la cresta cenomaniense del Cantal del Pixaor en la vertiente meridional sobre todo; y las astilladas y exiguas elevaciones como las del Maigmonet, o el Alt de la Ximenera.

Un ejemplo de la importancia del control estructural en la morfología de la ladera es el de la Serra del Frare hasta el Despenyador, y su continuación hasta la Serratella o Serra de Castalla; pues si la diferenciación entre cantil y talud en el tramo más septentrional (hasta el Despenyador), y del sector más oriental de dicha sierra, es además de litológica, top ográfica, se debe en gran medida a que morfoestructuralmente se trata de un monoclinal ligeramente inclinado a partir del escarpe; mientras que el tramo occidental de la Serra del Frare, es una cresta en la que sí existe diferenciación litológica, pero no sucede lo mismo topográficamente, y no existe la ruptura de pendiente tan acusada como en los otros sectores señalados (fig. 1). 


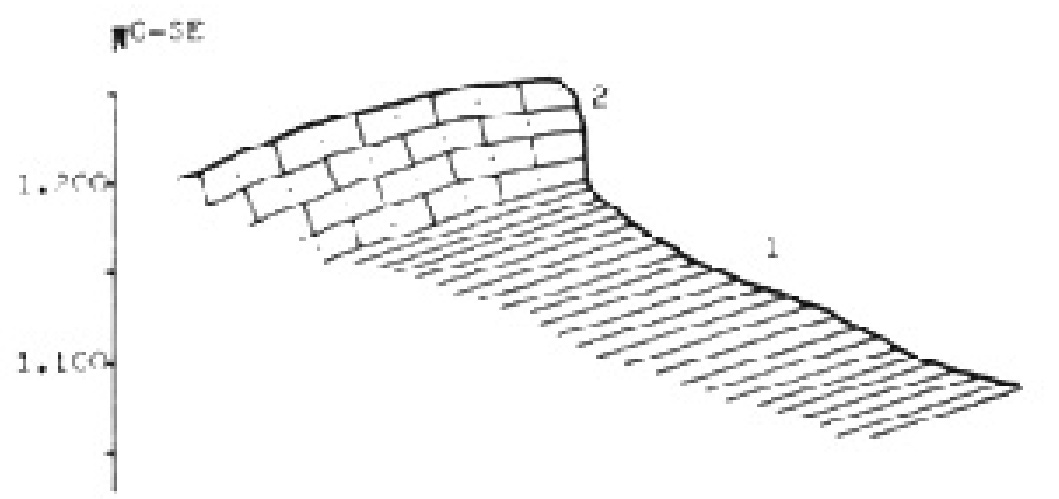

a)

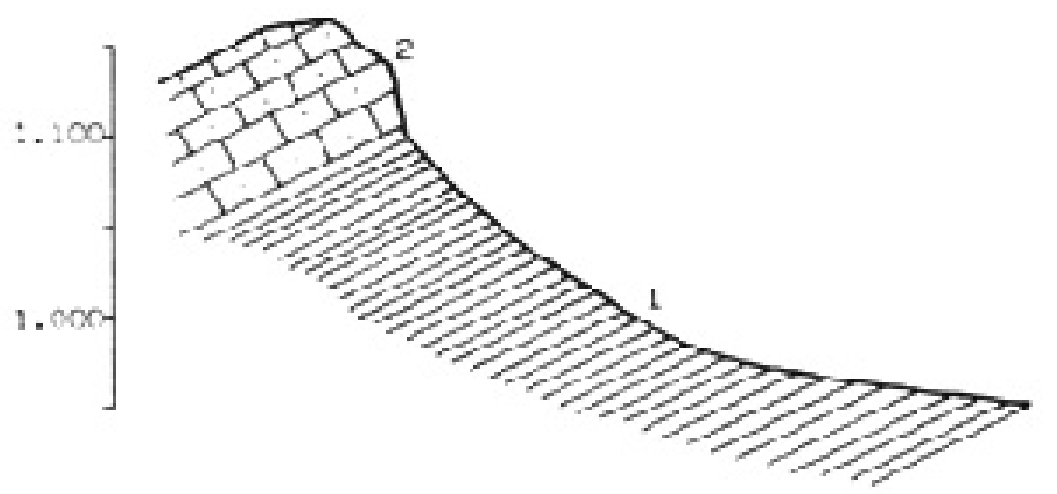

b)

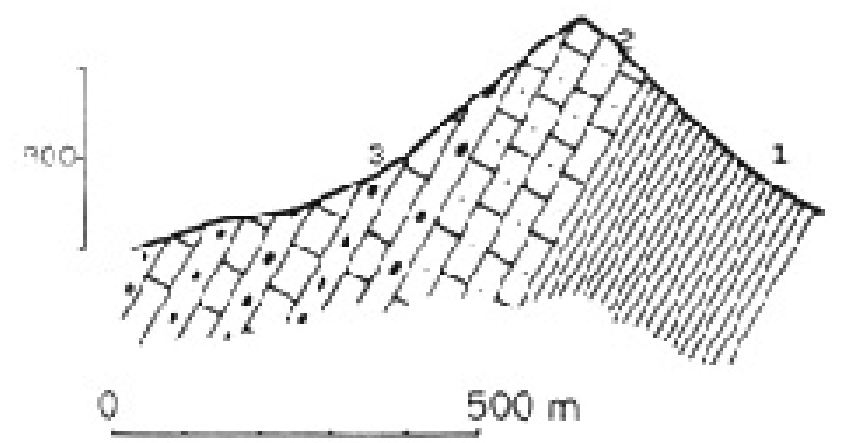

c)

FiguRA 1. Cortes de la ladera cantil talud de: a) sector inmediatamente al norte del Despenyador, b) Serra del Frare, sector oriental; c) Serra del Frare, sector occidental: 1. Margas langhienses. 2. Calcarenitas serravallienses. 3. Calcirruditas tortonienses. 
Sea del tipo que fuere, estas laderas, tienen una característica común que ya hemos señalado, se trata de escarpes de línea de falla o en su caso de los mismos espejos de falla (los cantiles), mientras que los taludes pueden responder a parte del bloque levantado que ha sido erosionado, o bien que la falla tiene allí un plano más oblicuo, o que se trate de taludes puramente erosivos.

De todos modos, el rasgo esencial de las mismas, es que se trata de unidades del relieve que confieren anfractuosidad al mismo, ya que en ellas, las pendientes generales son siempre superiores a $20^{\circ}$, o muy fuertes, por encima de $45^{\circ}$; pero no es sólo el valor de las pendientes el que nos permite hacer esta consideración, pues en sectores más bajos y acarcavados, estas pendientes también son frecuentes, pero en tramos muy cortos y sup erficies muy reducidas, son detalles del modelado, no como ocurre en este tip o de laderas en las que estas pendientes tienen un mayor desarrollo longitudinal y transversal, precisamente por tratarse de un hecho de raigambre estructural que constituye el verdadero armazón del relieve.

No es de extrañar por tanto que todas estas vertientes sean auténticos murallones que esquivan o rodean las vías de comunicación y en los que la más

\section{CUADRO DE PENDIENTES}

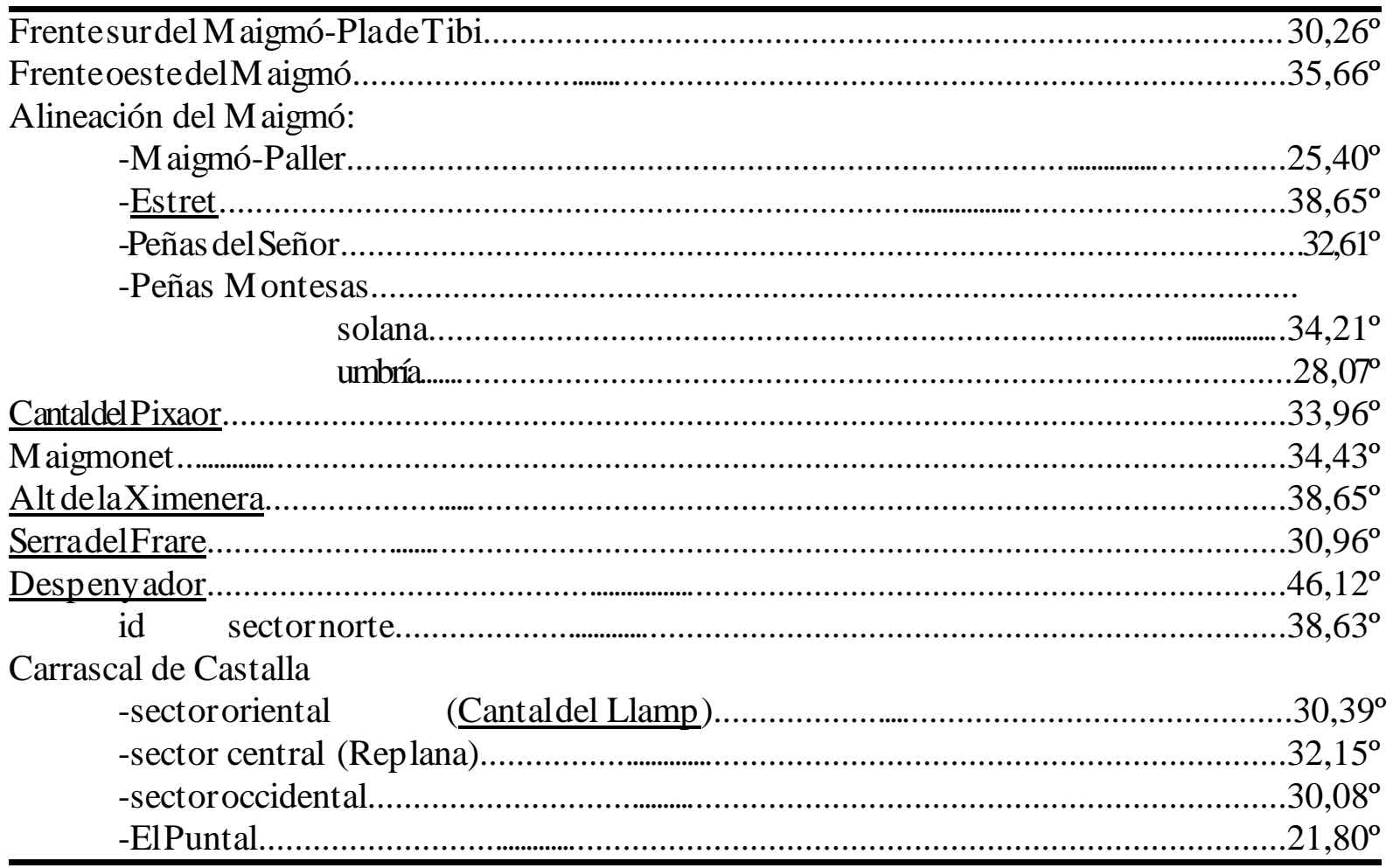


mínima rasgadura o descenso altitudinal adquiere singularidad por la importancia que han tenido para el habitante de un entorno en el que las sierras eran parte fundamental de su economía. Baste recordar alguno de estos topónimos como el Collao de les Fermoses entre la Rep lana y el Cantal del Llamp, el Collao de interrogante causal.

\section{Naturaleza y significación morfológica de las acumulaciones de ladera}

Las formaciones que se acumulan en las laderas consideradas presentan enormes diferencias que no se relacionan únicamente con su localización y, por ende, con la litología, sino que además existen distinciones que muy bien pueden derivar del ambiente en el que se sedimentó cada una de ellas.

Por la posición que ocupan en el talud, es obligado distinguir las formaciones de carácter eminentemente superficial, los canchales, que cronológicamente se les ha datado como actuales o subactuales: los llamados runars en el ámbito valenciano, tienen amp lia representación en las sierras, motivo por el cual han despertado gran interés entre los geógrafos.

En el Macizo del Maigmó también tienen buena representación otro tipo de acumulaciones como fenómeno del modelado cuya edad se aleja más en el tiempo y que debemos considerar p leistocenas no sólo p or su p osición relativa en los taludes, sino también por su textura, características morfométricas y sedimentológicas.

Se plantea de este modo una clara contraposición entre dos tipos de acumulaciones: los canchales, y los depósitos de ladera. Pero la realidad es mucho más compleja y con este sencillo esquema se obvian matices y precisiones que se observan en el campo y encuentran justificación en una serie de técnicas de análisis empleadas en el laboratorio.

\subsection{Los canchales: morfología superficial y perfiles}

Al contrario de lo que sucede con los depósitos de ladera más antiguos, la detección de los canchales a través de los trabajos fotogramétricos previos a los recorridos de campo es en cierto modo sencilla, sobre todo cuando representan las únicas áreas desprovistas de vegetación en una vertiente densamente poblada por ésta, sobre todo carrascas, que encuentran en estos ecótopos sus condiciones más idóneas, al menos en nuestro ámbito de estudio. No sucede lo mismo cuando la ladera es parca en vegetación, hecho que coincide puntualmente en las solanas.

Desde esta perspectiva, se puede definir como canchales a aquellas superficies con fuertes pendientes constituidas por derrubios, incluso bloques, libres -en sup erficie- de cualquier tipo de matriz fina, y en los que la vegetación prácticamente no existe, o al menos se trata de plantas de porte reducido, y a los que hay que conceder movilidad actualmente ${ }^{2}$. Estas acumulaciones de derrubios, es lógico pensar que se asocien a una fuente de alimentación caliza -los cantiles-, aunque puede suceder que esto no se dé en el momento actual. Con 
estas premisas, cabe esperar que el desarrollo de los canchales corra paralelo al de los cantiles, pero esto no sucede así, con lo que se plantea una primera interrogante causal.

De tratarse de un fenómeno ligado a un único sistema morfogenético, no encontraría justificación la discontinuidad esp acial a lo largo de un mismo talud. Es necesario pensar en una convergencia de procesos para su explicación ${ }^{3}$, pero sobre todo en un condicionante esencial: la preparación de una roca coherente para su desintegración mediante la tect onización. Esta premisa se comprueba cuando en no p ocas ocasiones los canchales se relacionan con los sectores más tectonizados de los cantiles. Responsabilizar sólo a fenómenos de clima frío en la génesis de los canchales resulta p or tanto absurdo, las precipitaciones de elevada intensidad, y los fenómenos de desprendimientos masivos de bloques adquieren may or responsabilidad en la formación de los mismos.

\subsubsection{Tipos de canchales}

Si se atiende a su forma perimetral debemos distinguir dos tipos que están directamente relacionadas con las dimensiones del cantil y la morfología del talud. En los dos tipos a los que se hace alusión la forma es alargada, pero mientras en un caso su disposición es transversal al escarpe, en el otro, ésta es longitudinal, paralela a las curvas de nivel.

Los del último tipo adquieren mediante la coalescencia de varios o múltiples semiconos de derrubios tal forma, y con ello una mayor continuidad espacial, precisamente la misma característica que se consideraba en el primer conjunto de laderas cantil-talud señaladas anteriormente. Así, este tipo de canchales calificados con el expresivo adjetivo de «mandiles» o «delantales» ${ }^{4}$ están presentes en la Serra del Frare, Despenyador y su continuación hasta la Serratella o Serra de Castalla; en El Puntal, y también en algunos sectores tanto de la solana como de la umbría de las Peñas Montesas, morfoestructura que si bien la hemos incluido en el segundo conjunto, por su continuidad considerable influye igualmente en la forma perimetral de los canchales que se desarrollan en el sopié de las calizas cenomanienses (fig. 2).

Por otra parte, los canchales alargados en sentido de la pendiente resp onden a dos causas fundamentales: la fuente de alimentación -cantil-, es de dimensiones muy reducidas, con frecuencia se trata de culminaciones muy exiguas y ap itonadas; o bien que dicha forma se debe a las condiciones top ográficas en las que se encuentren los taludes, tal es el caso de la existencia de pequeñas vaguadas, con lo que los canchales que tienen esta forma han recibido denominaciones muy varias: canchales «barranco» ${ }^{5}$, o «regueros de piedras $»^{6}$.

A la primera de las causas se asocian los canchales que hay al pie del Maigmonet, en el frente oeste del Maigmó propiamente dicho, en el Cantal del Pixaor, o en el Alt de la Ximenera. En ellos no es ap licable la denominación de canchales «barranco», pues ésta parece más propia de aquellos que rellenan parcialmente pequeñas vaguadas y que presentan un perfil transversal convexo. En estos casos, estas vaguadas se corresponden con pequeñas fallas o desgarres en los que la trituración es extrema, y donde con seguridad, los derrubios son 
«ordenados» por las lluvias torrenciales. Así sucede en algunos casos del frente norte de la alineación del Maigmó al oeste de dicho pico, en el frente meridional del mismo que domina el Pla de Tibi, en el frente occidental del Maigmonet, o en el Cantal del Pixaor (fig. 2).

\subsubsection{Perfiles de los canchales}

A los aspectos puramente superficiales, para la comprensión de este hecho, hay que recurrir también a un análisis de la naturaleza interna de los canchales, pues con ello comprobaremos que el ap arente caos sup erficial es sólo eso, una ap ariencia.

La may or dificultad estriba en no poder contar con cortes de todos los canchales, sólo se han podido analizar p or tanto los ejemplos que hay alrededor del Maigmonet, en el corte de una pista forestal que pasa por el Collao d'Exaú, otro más cercano a la culminación de dicho alto, y otro en el Cantal del Pixaor.

Del análisis de todos ellos se desprenden algunas consideraciones relacionadas con la dinámica de los canchales. En primer lugar destaca el hecho de que en profundidad hay cierta clasificación por tamaños de los derrubios, están como estratificados alternando cantos más gruesos con otros más pequeños o con abundancia de fracción fina. En los canchales de la ladera oeste del Maigmonet, este esquema se simplifica a un cubrimiento superficial de derrubios más groseros, seguidos en profundidad de otros de menor tamaño (fig. 3a), hecho que también se ha comprobado en dos de los cortes analizados.

En estos dos casos, muy próximos entre sí, existe una diferencia relacionada con el poblamiento vegetal y funcionalidad del canchal: uno de ellos, en sup erficie está completamente libre de vegetación, mientras que el otro está muy colonizado. En ambos, por debajo de los derrubios sueltos existe un «estrato» que destaca por su coloración (pardaoscura), con abundante materia orgánica, con una matriz fina areno-limosa y con gran desarrollo de las raíces de las plantas, no sólo de las que enraízan en el propio canchal como ocurre en el segundo de los casos citados, sino también de las carrascas que rodean estas formaciones. Es evidente por tanto que se hallan trabados por la vegetación (figs. 3b y c).

Por lo observado en el macizo, y en otros sectores del ámbito valenciano, esto sucede especialmente en las umbrías, donde el cubrimiento vegetal es muy denso, y en este caso concreto marca el carácter acusadamente progresivo de los carrascales en las sierras valencianas. En estos perfiles es evidente que la evolución de las vertientes tiende hacia su estabilización por parte de la biomasa que paraliza la migración de los derrubios en favor de las pendientes.

Sin embargo, es llamativo el hecho de que la colonización se encargue de paralizar primero las capas infrayacentes; pero no es un hecho casual. En los

FiguRA 2. Esquemas de la morfología perimetral de los canchales de: a) el Despenyador y sector oriental de la Serra del Frare; b) Frentes sur y oeste del Maigmó propiamente dicho, y frente norte de la alineación del Maigmó; c) Alt de la Ximenera; d) Maigmonet; e) Cantal del Pixaor y f) Peñas Montesas. 


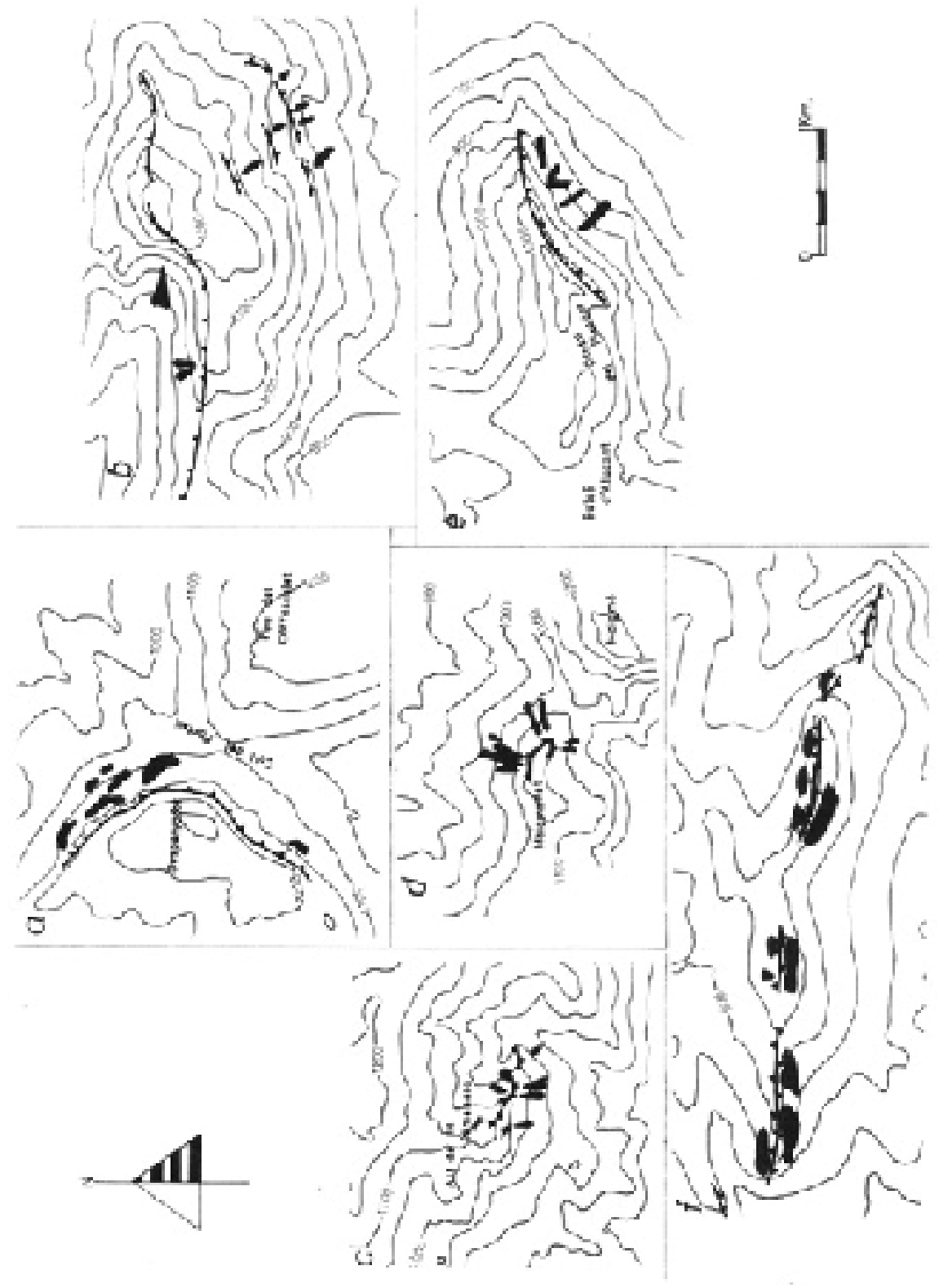


canchales en los que prácticamente no hay vegetación como en la ladera oeste del Maigmonet o la sur del Cantal del Pixaor, las variaciones granulométricas son parecidas a las apuntadas en los casos anteriores. En el ejemplo del Maigmonet, se analizó en su día un canchal «reguero» caracterizado por la presencia de derrubios suelt os en superficie, una capa muy delgada de material fino, y la roca in situ a continuación (fig. 3a); en el Cantal del Pixaor, se excavó un canchal para comprobar de qué modo variaban el tamaño y composición granulométrica, de tal manera que después de observar la alternancia de lechos según el tamaño, se comprobó que a medida que se profundizaba, aparecía fracción fina entre los derrubios. Exactamente igual que en algunos perfiles de canchales de la umbría del Maigmonet (figs. 3b y c).

No olvidemos que en otras ocasiones ya se han destacado los fenómenos de lixiviado en los canchales, y que éstos actuarían como medios de tamizaje in situ, que ante todo provocarían la migración en profundidad de la fracción fina. No es de extrañar pues, que en los sectores más propicios al crecimiento vegetal, éste busque en dichas condiciones top oecológicas el sustrato que pueda retener mejor la humedad y del que puedan extraerla (figs. 3b y c).

Perfil realmente significativo lo presenta un depósito situado en la umbría del Maigmonet, con orientación NE a 1.024 m.s.n.m. (perfil UM). Se trata de un canchal completamente colonizado p or la vegetación en cuy o corte resaltan dos niveles de suelo turboso con espesores de 30 y $13 \mathrm{~cm}$, separados entre sí y la sup erficie por tres niveles de canchal (fig. 4). En ellos, y al respecto del lixiviado, destaca el incremento del porcentaje de materia fina a medida que consideramos niveles sucesivamente más inferiores.

Los tres niveles analizados presentan grandes analogías en la granulometría y morfología de los sedimentos, de tal manera que la distribución porcentual de las tres fracciones (gruesa, grava y fina), muestra fehacientemente el marcado predominio de la primera. A ello hay que añadir que dentro de la fracción gruesa, el valor modal de los tamaños de los cantos coincide en las tres muestras (entre 2’01 y 3’00 cm), salvo la excepción de la muestra I UM en la que comparte idéntico porcentaje con el intervalo inmediatamente sup erior. Es pues un rasgo común el tamaño de los cantos, ya que todos los casos, más de tres cuartas partes del número total de derrubios son iguales o inferiores a $5 \mathrm{~cm}$. En cuanto a la morfología, es de destacar que en las tres muestras, la aplicación del índice de aplanamiento de Tricart y Cailleux ${ }^{7}$, depara valores medios muy próximos, alrededor de 2’5. Se puede constatar a través de todos estos rasgos que la semejanza de los tres niveles en la fracción gruesa es patente y significativa, pues se trata de la fracción predominante y que confiere may or singularidad a estos depósitos.

Naturalmente, existen diferencias, pero son mínimas, al menos en esta fracción. Pues en lo que respecta a los resultados de los análisis de la fracción fina, éstos desvelan algunos matices no carentes de interés. Ya se ha señalado que la matriz fina aumenta en el porcentaje de la muestra a medida que ésta está a may or profundidad. Pero en la granulometría específica de esta fracción, las arenas son cada vez más frecuentes en el mismo sentido del perfil en detrimento de los limos y las arcillas. En cuanto a la distribución frecuencial de las arenas 

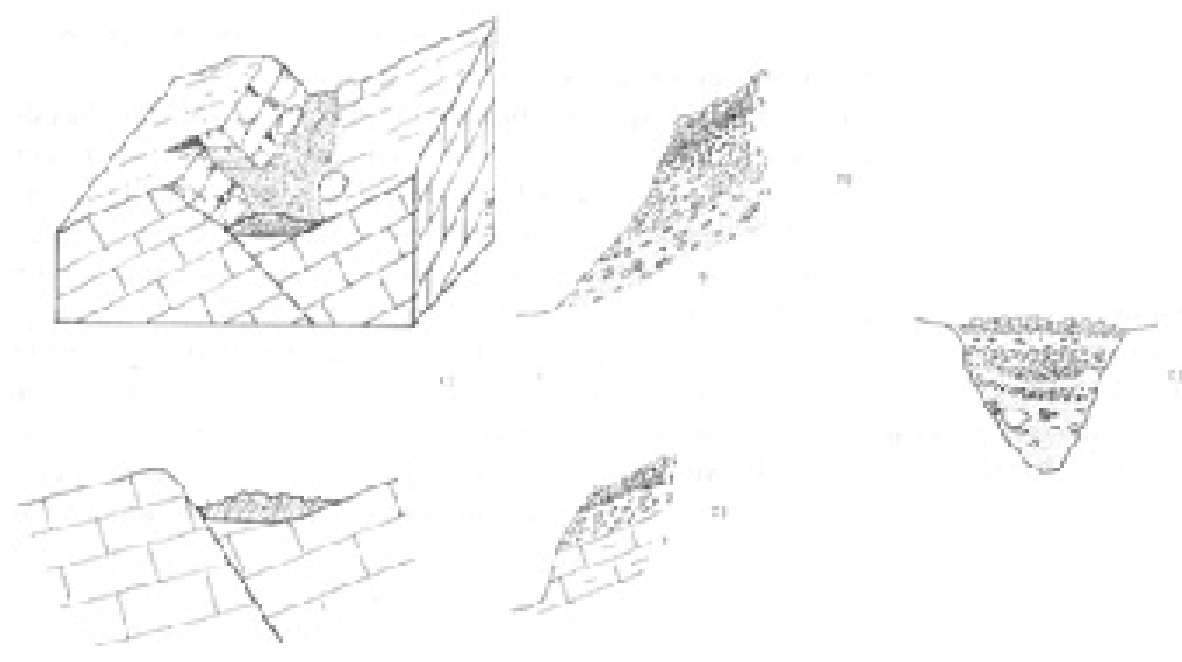

Figura 3. A) Bloque diagrama y corte transversal de canchal «barranco» al oeste del Maigmonet. B) Perfil de la pista forestal del Collao d’Exaú (este); B1: depósito de solifluxión pleistocena; B2: costra calcárea, B3: derrubios con abundante matriz fina de color negruzco; B4: derrubios con matriz fina negruzca y tupida red de raíces. B5 y 6: derrubios sueltos superficiales; c) perfil de la pista forestal del Collao d'Exaú (este), C1: Calizas fétidas miocenas, C2: derrubios con matriz fina y abundantes raíces, C3 y 4: derrubios sueltos superficiales colonizados parcialmente por la vegetación; D) derrubios ordenados en cárcava preexistente, pista del Collao d'Exaú (oeste).

por tamaños, hay una clara diferencia entre las muestras I y III UM frente a la V UM. Si en las dos primeras no hay ningún tipo de selección y en sus respectivos histogramas de frecuencias hay un mínimo central entre dos picos, en la tercera muestra, la curva que representase las frecuencias sería comp letamente opuesta. Por otra parte, los histogramas de las dos primeras son muy semejantes, al menos en sus trazas -los picos tienen ubicación muy varia-, a los de otras muestras analizadas en otra ocasión en acumulaciones de ladera también en el Maigmó ${ }^{8}$.

Se trata portanto detres formaciones de naturaleza semejante, superp uestas estratigráfica $\mathrm{y}$, por ende, cronológicamente. Actuales o subactuales, en condiciones que en los dos primeros niveles son prácticamente iguales. De tal manera que se pueden interpretar como tres canchales sucesivos que han evolucionado a partir de un mismo cantil y en condiciones ambientales muy semejantes en las que la vegetación ha desempeñado un papel fundamental en la fijación de cada masa por su base, todo lo cual no significa que se impidan por completo movimientos ligados al cizallamiento entre las capas (shearing de la bibliografía anglosajona) citados para el caso de derrubios ordenados (eboulis ordenés) por el Dr. Rossello Verger ${ }^{9}$. Aspecto de tal formación tienen dep ósitos como los que hemos visto anteriormente, y otros localizados al oeste del Collao 
d'Exaú que yacen sobre otras acumulaciones más antiguas regularizando un modelado más antiguo de cárcavas sobre margas (fig. 3d).

También se ha podido observar que los perfiles transversales de los taludes, son un factor imp ortante en la naturaleza de los canchales, y a que los mayores espesores dependen no sólo del salto, sino también de la top ografía transversal del talud. De esta forma, los taludes que presentan vaguadas -estructurales o erosivas-, al ser receptores de derrubios desprendidos, éstos alcanzan may ores espesores, como en el caso de la ubicación de la muestra UM. Pero no es esta condición indispensable, ya que en otros sectores, esto también se cumple e infrayacentes a estas formaciones actuales o subactuales -holocenas-, hay otras que por su coloración -rojiza, frente a coloraciones gris claro o negruzcos de los holocenas- y por la existencia de una discontinuidad entre ambos depósitos como es un encostramiento calcáreo, tenemos que convenir en que son más antiguas, pleistocenas (fig. 3b).

Otro de los rasgos diferenciadores es la cementación total o parcial de los sedimentos. Por debajo de las formaciones que hemos considerado holocenas, en discordancia erosiva o no, se han observado encostramientos calcáreos ya sea en el límite superior o techo del depósito pleistoceno, o bien afectando a todo el depósito. En la primera de estas situaciones, un aspecto llamativo son las abundantes inflorescencias de carbonato cálcico que afecta a estos materiales; mientras que en la segunda se ha podido comprobar que la cementación ni es total ni completamente coherente, ya que en los cortes en los que se aprecia está fuertemente meteorizada.

En las que hemos denominado acumulaciones de solifluxión, la característica principal es el marcado predominio de crioclastos dentro de la fracción gruesa, de las llamadas esquirlas de estallido. Pero también en estos sedimentos de solifluxión cabe la posibilidad de establecer distinción entre dos conjuntos: la solifluxión, entendiendo como tal a una masa en la que a pesar de su considerable espesor no se aprecia estrat ificación alguna; y la solifluxión estratificada (grèzes litées), donde alternan lechos de materiales más o menos finos, siendo este el aspecto que los singulariza.

La solifluxión en el sentido que le hemos otorgado al término, es el más abundante, y tanto en la pista que sube al Collado d'Exaú, como la carretera que sube al Balcó d'Alacant, como en la parte este del Barranc de l'Estret, o en el Portell, tienen considerable extensión.

Por contra, la solifluxión estratificada está bien localizada a ambos lados del Collao d'Exaú, pero resultan muy interesantes las de su lado oeste, ya que la vegetación, menos abundante no nos impide su análisis. Se trata de una masa de gelifractos estratificados entre lechos de material más fino, con predominio del arcilloso en la que no faltan grandes bloques.

\subsection{Las acumulaciones de ladera pleistocenas}

Además de la cronología, el rasgo esencial que diferencia a éstas de los canchales es su mayor espesor, y por tanto mayor importancia en el modelado de las vertientes; pero también es preciso señalar que en ellas se ha visto en las 

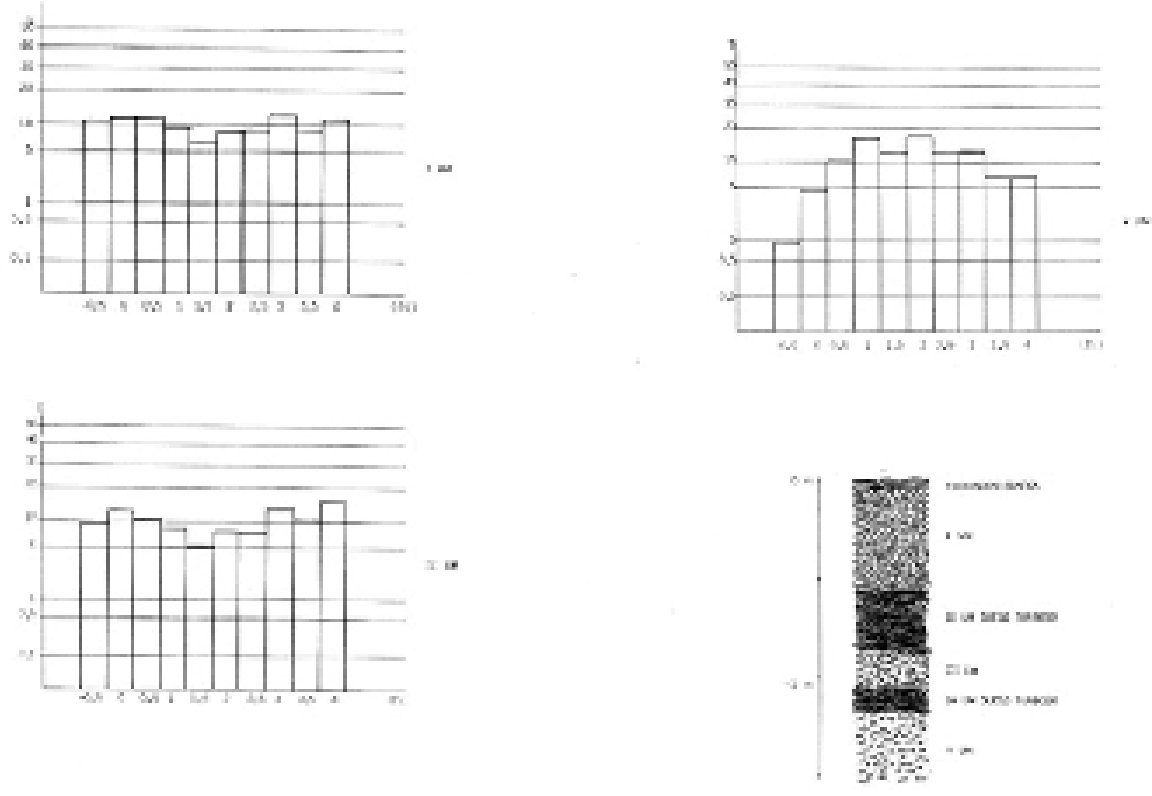

FIGURA 4. Estratificación e histogramas de frecuencias de las arenas del perfil UM. 
sierras mediterráneas, una de las manifestaciones más claras del modelado derivado de climas fríos, y en especial a las pulsaciones frías pleistocenas ${ }^{10}$.

Pero no todos los depósitos que aparecen infray acentes a los canchales, o de clara edad pleistocena, deben su origen a condiciones de clima frío. Por esta razón, es conveniente precisar que hay una clara diferencia entre las que podemos denominar acumulaciones o depósitos de solifluxión y los depósitos de ladera sensu lato. En estos últimos, con material grosero muy heterométrico, existen además de derrubios de clara raigambre crioclástica, es decir, auténticas esquirlas de gelifracción, gran abundancia de cantos e incluso bloques. Todo ello, tanto en estos depósitos como en los de solifluxión, envuelto por una matriz fina más abundante que en el caso de los canchales que hemos analizado anteriormente, y de coloración rojiza.

Ya se ha señalado que uno de los rasgos que los diferencia de los sedimentos holocenos era su coloración que les confiere la matriz fina, sin duda resultado del arrastre de arcillas de descalcificación (terra rossa) procedente de los terrenos calcáreos carsificados situados ladera arriba. Las características granulométricas más importantes de estas «paleoformas» (como las denominamos en otra ocasión), si bien presentan diferencias apreciables con los canchales analizados ahora, es de señalar también que en sus histogramas de frecuencias y en los tamaños modales existen marcadas semejanzas ${ }^{11}$.

\subsection{Significación morfológica de canchales y acumulaciones de ladera más antiguas}

Ambos sedimentos, juntos o por sep arado, en una primera ap roximación son fenómenos que se encargan de regularizar las pendientes, ya que en un esquema primigenio de cualquier ladera cantil-talud previo a la aparición de cualquier formación en el talud, presentaría pendientes superiores a las resultantes de su evolución posterior.

Ya se ha señalado que la morfología de estas laderas tiene una marcada raigambre morfoestructural derivada de una tectónica de fractura que se ha encargado de configurar de forma más decisiva el relieve de todo el macizo. Después, en época probablemente pliocena, tuvo lugar la fase de disección princip al, que si bien no fue muy larga, sí fue muy efectiva ${ }^{12}$. Las formas que tratamos son posteriores a esos momentos de la evolución del macizo, ya que por un lado existen depósitos que se ciñen perfectamente a las líneas de falla, y se encargan de suavizar la p endiente estructural -p róxima a la vertical- de un salto de falla. Pero si esta labor corresponde en un primer lugar a las acumulaciones antiguas, en estas laderas compuestas por materiales detríticos, aparecen canchales actuales o subactuales que continúan con la tendencia iniciada por aquéllas de regularizar las pendientes.

También se ha observado que ambos sedimentos yuxtapuestos, con o sin discontinuidad aparente, están colmatando cárcavas p reexistentes en los taludes sobre materiales margosos, vestigios de un relieve más anfractuoso que el actual y producto de la fase de disección principal, o incluso vaguadas de dimensiones may ores como en el lado este de la subida al Collao d'Exaú. 
Entre las acumulaciones antiguas, y los canchales actuales o subactuales, hay una costra calcárea que afecta a la parte superior de los depósitos de solifluxión o derrubios de ladera, o bien a todo el depósito infray acente, claramente pleistoceno. En éstos ha sido en los que se ha observado deformaciones que bajo ningún concepto se pueden atribuir a la actuación del hielo, ya que asoma entre la solifluxión el Keuper; se trata de afloramientos calificados por el Dr. García Fernández como flamígeros ${ }^{13}$ que inclinan estos depósitos. Circunstancia que ha sido constatada tanto en la carretera que sube al Balcó d'Alacant, como en el camino que desde ésta sube al Callao d'Exaú. Son pues, claras manifestaciones de la neotectónica que en época pleistocena ha terminado de configurar las morfoestructuras del macizo.

Con todo, se puede concluir diciendo que todas estas acumulaciones de ladera tienen el mismo significado morfográfico como atenuadoras de un relieve morfoestructural en unos casos y erosivo en otros, que sin duda era más anfractuoso de lo que es actualmente, que como hemos p odido ap reciar en el cuadro de pendientes generales de las laderas, éstas y a son considerables.

Este fenómeno de regularización de las pendientes, como elemento de la evolución de las vertientes, no es homogéneo, y las discontinuidades esp acio-temporales propias de las formas heredadas, están también presentes en los canchales actuales o subactuales. En el presente, ambas formas de acumulación, pierden localmente tal condición, y con el concurso de otros procesos, fundamentalmente la erosión lineal, participan de la dinámica del modelado del macizo.

Esta conexión entre unos procesos y otros se realiza de forma muy diversa, y de ello depende la movilidad o estatismo de buena parte de estas laderas. Un caso expresivo de acumulaciones antiguas afectadas por el encajamiento actual de la red de avenamiento lo constituyen los abarrancamientos, incluso cárcavas (en el sentido más amplio, pues se han observado hasta fenómenos de piping) que se encargan de lacerar aunque moderadamente los depósitos de solifluxión de la parte alta del ramal oriental del barranco que desagua por el Estret.

Evidente es la conexión existente entre los llamados anteriormente canchales «barranco» y la red de barrancos y ramblas, sobre todo en los que por la parte oeste del Maigmonet, sur del Maigmó, frente norte de la alineación del Maigmó al oeste de dicho pico, Alt de la Ximenera, y Cantal del Pixaor: unos de forma más directa, otros menos, todos acaban por suministrar material grosero a las respectivas cuencas vertientes.

No sucede lo mismo con los canchales y acumulaciones de solifluxión de la ladera este del Maigmonet (umbría), umbría del Maigmó propiamente dicho y sector del Despenyador. En ellos, el espeso manto vegetal que coloniza casi por completo las acumulaciones más antiguas, y de forma marginal a los canchales, es una traba a los procesos erosivos. Por el contrario, en dichas áreas es de resaltar los procesos de pedogénesis. Sin embargo, no quedan libres de erosión únicamente las laderas bien cubiertas por la vegetación, aquellas que, compuestas por depósitos de solifluxión o de derrubios indiferenciados, con un poblamiento vegetal más escaso, por sus propias condiciones texturales (muy permeables y con grandes facilidades de infiltración), o por mediar una protección 
física en forma de encostramiento calcáreo, presentan escasos síntomas de erosión por no decir nulos. Sólo si median factores externos ésta hace acto de aparición mediante la incipiente incisión de regueros o pequeñas cárcavas tal y como sucede con la solifluxión de Penyes Roges que ha visto alteradas sus condiciones en el momento en el que se han elaborado terracillas para repoblar pinos. Lo mismo sucede en algunos sectores de la solana de Peñas Montesas y en la Serra del Frare a exp ensas de desmontes para caminos, o para parcelaciones de una urbanización de Petrer, el Palomaret. De estas modificaciones tampoco están libres las acumulaciones situadas en las umbrías, ya que en el caso de la pista que desde la carretera al Balcó d'Alacant asciende al Collao d'Exaú, las capas superficiales de derrubios se muestran especialmente activas, como prueba de su origen y naturaleza. 


\section{APÉNDICE}

\section{MUESTRA I UM}

$\begin{array}{rrrr}\text { Fracción gruesa } & 1,189 \text { gr } & 89,7 \% \\ \text { id grava } & 68,22 \text { gr } & 5,1 \% \\ \text { id fina } & 68,28 \text { gr } & 5,2 \%\end{array}$

*Análisis Fracción gruesa

$\begin{array}{clrr}\text {-Granulometría } & \text { tamaño }(\mathrm{cm}) & \mathrm{n}^{\circ} \text { cantos } & \% \\ & 1,01-2 & 11 & 20,00 \\ 2,01-3 & 12 & 21,82 \\ 3,01-4 & 12 & 21,82 \\ 4,01-5 & 8 & 14,54 \\ 5,01-6 & 3 & 5,45 \\ 6,01-7 & 6 & 10,91 \\ 7,01-8 & 2 & 3,63 \\ 10,01-11 & \underline{1} & 1,82\end{array}$

- Indice de aplanamiento $\overline{\mathrm{Ai}}=2,68$

*Análisis Fracción Fina

Arenas 11,95\%

Limos y arcillas 88,05\%

-Granulometría de las arenas

\begin{tabular}{lcr} 
tamaño (fi) & peso (gr) & \multicolumn{1}{c}{$\%$} \\
$-0,5$ & 0,905 & 11,09 \\
0 & 0,927 & 11,36 \\
0,5 & 0,941 & 11,53 \\
1 & 0,750 & 9,19 \\
1,5 & 0,514 & 6,29 \\
2 & 0,723 & 8,86 \\
2,5 & 0,700 & 8,58 \\
3 & 1,030 & 12,62 \\
3,5 & 0,718 & 8,80 \\
4 & 0,952 & 11,66 \\
& 8,160 &
\end{tabular}


MUESTRA III UM

$\begin{array}{crr}\text { Fracción gruesa } & 839,3 & 76,9 \% \\ \text { Fracción grava } & 105,0 & 9,6 \% \\ \text { id fina } & 146,2 & 13,4 \%\end{array}$

*Análisis Fracción gruesa

$\begin{array}{cl}\text {-Granulometría } & \text { tamaño(cm) } \\ & 1,01-2 \\ & 2,01-3 \\ & 3,01-4 \\ & 4,01-5 \\ 5,01-6 \\ 9,01-10 \\ 10,01-11\end{array}$

$\begin{array}{cc}\mathrm{n}^{\mathrm{o}} \text { cantos } & \% \\ 12 & 19,67 \\ 25 & 40,98 \\ 15 & 24,59 \\ 4 & 6,56 \\ 3 & 4,92 \\ 1 & 1,64 \\ \frac{1}{64} & 1,64\end{array}$

- Indice de aplanamiento $\overline{\mathrm{Ai}}=2,41$

* Análisis Fracción Fina

Arenas 25,7\%

Limos y arcillas 74,3\%

-Granulometría de las arenas

\begin{tabular}{lcr} 
tamaño (fi) & peso (gr) & \multicolumn{1}{c}{$\%$} \\
$-0,5$ & 2,361 & 9,18 \\
0 & 3,142 & 12,22 \\
0,5 & 2,685 & 10,44 \\
1 & 2,069 & 8,05 \\
1,5 & 1,382 & 5,37 \\
2 & 2,045 & 7,95 \\
2,5 & 2,006 & 7,80 \\
3 & 3,354 & 13,05 \\
3,5 & 2,676 & 10,41 \\
4 & 3,988 & 15,51 \\
& 25,708 &
\end{tabular}




\section{MUESTRA V UM}

$\begin{array}{rcc}\text { Fracción gruesa } & 932 \text { gr } & 75,9 \% \\ \text { id grava } & 66,5 \text { gr } & 5,4 \% \\ \text { id fina } & 228,5 \text { gr } & 18,6 \%\end{array}$

*Análisis Fracción gruesa

$\begin{array}{cl}\text {-Granulometría } & \text { tamaño(cm) } \\ & 1,01-2 \\ & 2,01-3 \\ & 3,01-4 \\ 4,01-5 \\ 5,01-6 \\ 6,01-7 \\ 9,01-10\end{array}$

$\begin{array}{cc}\mathrm{n}^{\mathrm{o}} \text { cantos } & \% \\ 9 & 16,66 \\ 15 & 27,78 \\ 10 & 18,52 \\ 12 & 22,22 \\ 3 & 5,55 \\ 4 & 7,41 \\ \frac{1}{54} & 1,85\end{array}$

- Indice de aplanamiento $\overline{\mathrm{Ai}}=2,46$

* Análisis Fracción Fina

Arenas 42,01\%

Limos y arcillas 57,99\%

-Granulometría de las arenas

$\begin{array}{lcc}\text { tamaño (fi) } & \text { peso (gr) } & \% \\ -0,5 & 0,402 & 9,6 \\ 0 & 1,855 & 4,41 \\ 0,5 & 4,221 & 10,05 \\ 1 & 6,957 & 16,15 \\ 1,5 & 5,152 & 12,26 \\ 2 & 7,162 & 17,04 \\ 2,5 & 5,115 & 12,17 \\ 3 & 5,407 & 12,87 \\ 3,5 & 2,886 & 6,88 \\ 4 & 2,859 & 6,80 \\ & \text { 42,016 } & \end{array}$




\section{NOTAS BIBLIOGRÁFICAS}

1 MARCO MOLINA, J. A. El Macizo del Maigmó. Estudio de Geografía Física. Inst. de Est. «Juan Gil Albert». Alicante, 1987, pp. 92-115.

2 Ibídem. Op. cit., pp. 98-101.

3 ROSSELLO VERGEL, V. M. «Los canchales de montaña calcárea y los factores termoclásticos». Actas de la II Reunión Nacional del Grupo Español de Trabajo del Cuaternario. Jaca, 15-20 septiembre 1975. Ed. Alberdi, M. T. et alteri, Madrid, 1977, p. 225.

4 Ibídem. «Los canchales...». Op. cit., p. 225.

5 Ibídem. «Los canchales...». Op. cit., p. 226.

6 LÓPEZ ONTIVEROS, A., y MORALES GIL, A. «Los derrubios de ladera en la sierra de 'El Carehe' (Murcia)». Actas de la II Reunión Nacional del Grupo Español de Trabajo del Cuaternario. Jaca, 15-20 septiembre 1975. Ed. Alberdi, M. T. et alteri. Madrid, 1977, p. 139.

7 KÖSTER, E. y LESER, H. Geomorphologie I. BodenkundlicheMethoden. Morphometrie und Granulometrie. Braunschweig, G. Westermann, 1967, p. 70.

8 MARCO MOLINA, J. A. Op. cit., p. 103.

9 ROSSELLO VERGEL, V. M. «Los canchales...». Op. cit., p. 226.

10 Ibídem. «Clima y morfología pleistocena en el litoral mediterráneo español». Papeles del Departamento de Geografía de Murcia, II, 1970, pp. 79-108.

11 MARCO MOLINA, J. A. Op. cit., pp. 102-109.

12 GARCÍA FERNÁNDEZ, J. El Maigmó (Análisis Morfoestructural), 1988.

13 Ibídem. Op. cit. 